\title{
Independent component analysis applied to pulse oximetry in the estimation of the arterial oxygen saturation (SpO2) - a comparative study
}

\author{
Jensen, Thomas; Duun, Sune Bro; Larsen, Jan; Haahr, Rasmus Grønbek; Toft, M. H.; Belhage, B.; \\ Thomsen, Erik Vilain
}

Published in:

Annual International Conference of the IEEE Engineering in Medicine and Biology Society, 2009. EMBC 2009

Link to article, DOI:

10.1109/IEMBS.2009.5333746

Publication date:

2009

Document Version

Publisher's PDF, also known as Version of record

Link back to DTU Orbit

Citation $(A P A)$ :

Jensen, T., Duun, S. B., Larsen, J., Haahr, R. G., Toft, M. H., Belhage, B., \& Thomsen, E. V. (2009). Independent component analysis applied to pulse oximetry in the estimation of the arterial oxygen saturation (SpO2) - a comparative study. In Annual International Conference of the IEEE Engineering in Medicine and Biology Society, 2009. EMBC 2009 (pp. 4039-4044). IEEE. https://doi.org/10.1109/IEMBS.2009.5333746

\section{General rights}

Copyright and moral rights for the publications made accessible in the public portal are retained by the authors and/or other copyright owners and it is a condition of accessing publications that users recognise and abide by the legal requirements associated with these rights.

- Users may download and print one copy of any publication from the public portal for the purpose of private study or research.

- You may not further distribute the material or use it for any profit-making activity or commercial gain

- You may freely distribute the URL identifying the publication in the public portal 


\title{
Independent Component Analysis Applied to Pulse Oximetry in the Estimation of the Arterial Oxygen Saturation $\left(\mathrm{S}_{\mathrm{p}} \mathrm{O}_{2}\right)$ - a Comparative Study
}

\author{
Thomas Jensen, Sune Duun, Jan Larsen, Rasmus G. Haahr, Mette H. Toft, Bo Belhage, and Erik V. Thomsen
}

\begin{abstract}
We examine various independent component analysis (ICA) digital signal processing algorithms for estimating the arterial oxygen saturation $\left(\mathrm{S}_{\mathrm{p}} \mathrm{O}_{2}\right)$ as measured by a reflective pulse oximeter. The ICA algorithms examined are FastICA, Maximum Likelihood ICA (ICAML), Molgedey and Schuster ICA (ICAMS), and Mean Field ICA (ICAMF). The signal processing includes pre-processing bandpass filtering to eliminate noise, and post-processing by calculating the $S_{p} O_{2}$. The algorithms are compared to the commercial state-of-theart algorithm Discrete Saturation Transform (DST) by Masimo Corporation. It is demonstrated that ICAMS and ICAMF perform up to $13 \%$ better than DST. PPG recordings are done with a reflective pulse oximetry sensor integrated in an Electronic Patch. This system is intended for patients with chronic heart and lung conditions.
\end{abstract}

\section{INTRODUCTION}

Pulse oximeters intended for long-term monitoring of people living an everyday life with a chronic condition offer several challenges: 1) Design and construction of small, discrete, and low-power pulse oximeter devices. 2) Digital signal processing of photoplethysmograms (PPG) which are affected by the patient's motions, a less optimal monitoring site for the purpose of discreteness and long-term compatibility, and battery powered Light Emitting Diodes (LEDs). 3) Integration of these new technologies which typically features telehealth solutions into the established health care system.

We have previously reported the design and development of an Electronic Patch with integrated reflective pulse oximetry based on a novel ring-shaped optical sensor [1], [2], [3]. The Electronic Patch is shown in Fig. 1. All PPG measurements reported in this paper are performed with the Electronic Patch.

Estimating the $\mathrm{S}_{\mathrm{p}} \mathrm{O}_{2}$ from PPG data which are heavily distorted by noise and motion artefacts can be done by

This work was partly supported by the Danish Ministry of Science, Technology, and Innovation.

T. Jensen and J. Larsen are with the Technical University of Denmark, Department of Informatics and Mathematical Modeling, Richard Petersens Plads B321, 2800 Kgs. Lyngby, Denmark (Phone: + 45 45253923; Fax: +45 45872599; e-mail: jl@imm.dtu.dk).

S. Duun, R. G. Haahr and E. V. Thomsen are with the Technical University of Denmark, Department of Micro and Nanotechnology, Oersted Plads building 345 east, 2800 Kgs. Lyngby, Denmark

M. H. Toft and B. Belhage are with the University of Copenhagen, Bispebjerg Hospital, Bispebjerg Bakke 23, 2400 Copenhagen NV, Denmark

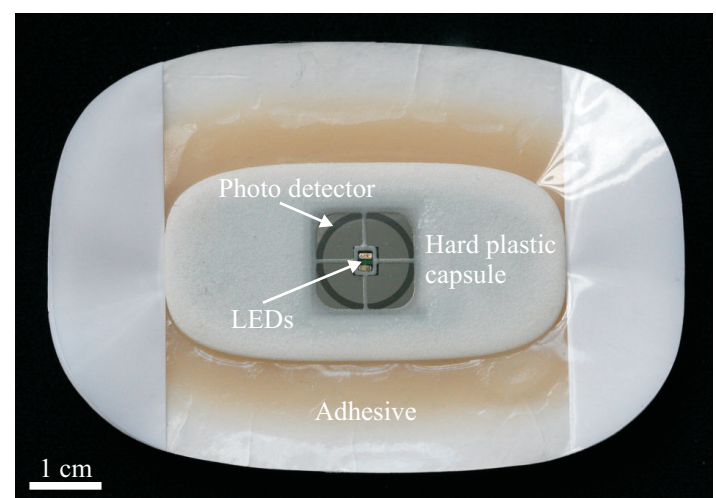

Fig. 1. The Electronic Patch. The patch contains a micro fabricated ringshaped silicon backside photodiode with a through chip hole for commercial surface mounted LEDs. This is packaged inside a hard plastic capsule with analog, digital, and RF electronics. The capsule is embedded in adhesive material as shown in the figure. The patch measures $88 \mathrm{~mm}$ by $60 \mathrm{~mm}$ and is $5 \mathrm{~mm}$ thick. The weight is 15.9 grams.

advanced digital signal processing. The commercial stateof-the-art, Discrete Saturation Transform (DST) by Masimo Corporation [4], [5], is often used in clinical pulse oximeters. To benefit most from the above mentioned category of lowpower pulse oximeters algorithms which are better at noise and motion filtering are needed.

Signal processing of PPG data using independent component analysis (ICA) has been reported by several authors [6], [7] as a promising technique for motion artefact reduction. These papers do not describe the problem of estimating the $\mathrm{S}_{\mathrm{p}} \mathrm{O}_{2}$ nor do they present a comparison with the DST algorithm. In this paper we evaluate four different ICA algorithms with respect to estimating $\mathrm{S}_{\mathrm{p}} \mathrm{O}_{2}$ from PPG data recorded on resting subjects (i.e. not motion distorted) but recorded with the Electronic Patch under low-power conditions (the LED driving current is $5 \mathrm{~mA}$ ). It is known that data is perturbed with electronic noise and the signal to noise ratio is low due to the low LED driving current. A sample of a raw PPG recording is shown in Fig. 2. The ICA algorithms are compared to the DST algorithm. Data and Matlab functions are online available from [8]

Most authors focus on the FastICA algorithm [9], [10], but there are several other ICA algorithms such as Maximum Likelihood ICA (ICAML) [11], [12], [13], Molgedey and Schouster ICA (ICAMS) [12], [14], [15] and Mean Field ICA 


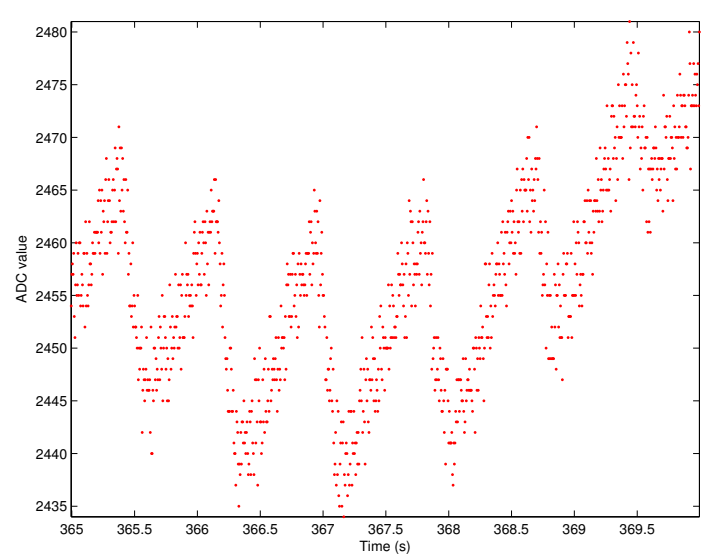

Fig. 2. A five seconds section of raw PPG data. The signal is noisy but the pulsating nature due to the heart beat is easily recognized.

(ICAMF) [16], [17]. The various ICA algorithms are based on different assumptions regarding the statistical properties of the source signals and works by optimizing different parameters such as autocorrelation and probability distributions in the attempt to separate sources and noise signals.

The hypothesis of this paper is that the quality of the separated PPG signals and consequently the $\mathrm{S}_{\mathrm{p}} \mathrm{O}_{2}$ prediction depends critically on the choice of ICA algorithm. Motivated by this we present a comparison of the mentioned four different ICA algorithms and the DST algorithm. We evaluate the algorithms by comparing the estimated $\mathrm{S}_{\mathrm{p}} \mathrm{O}_{2}$ values with reference values obtained by a commercial pulse oximeter for the intensive care unit.

\section{Discrete SATURATion Transform}

DST is based on the assumption that the measured signals can be described as a sum of a desired PPG signal $s(t)$ and a noise signal $n(t)$ due to motion artifacts. Masimo [4] defines the system as

$$
\begin{aligned}
x_{i r}(t) & =s(t)+n(t) \\
x_{r}(t) & =r_{a} s(t)+r_{v} n(t)
\end{aligned}
$$

with $x_{i r}(t)$ and $x_{r}(t)$ being the signals from the infrared and red recordings respectively and $r_{a}$ is the arterial ratio corresponding to the arterial saturation and $r_{v}$ is the ratio of venous, or non-arterial, components. The method is based on removal of the noise term $n(t)$ by the use of Adaptive Noise Cancelation (ANC) [18]. ANC requires a reference of the noise signal which is similar to the undesired noise signal. Such a reference noise signal is not available from pulse oximetry and Masimo therefore defines a reference noise signal $n^{\prime}(t)$ as a weighted difference of the normalized measured signals

$$
n^{\prime}(t)=x_{r}(t)-r x_{i r}(t) .
$$

By inserting (1) and (2) in (3)

$$
\begin{aligned}
n^{\prime}(t) & =r_{a} s(t)+r_{v} n(t)-r(s(t)+n(t)) \\
& =\left(r_{a}-r\right) s(t)+\left(r_{v}-r\right) n(t) .
\end{aligned}
$$

It is seen from (5) that when $r_{a}=r$ the reference noise signal $n^{\prime}(t)$ only contains the weighted noise of the measurements. The measured signal from the red LED $x_{r}(t)$ is applied as the input to the ANC with the reference signal $n^{\prime}(t)$ and the desired output of the ANC is the noise free PPG-signal $s(t)$. The coefficient $r$ is found iteratively by trying all values of $r$ corresponding to all $\mathrm{S}_{\mathrm{p}} \mathrm{O}_{2}$ levels from $0-100 \%$ in steps of e.g. $1 \%$. The power of the output signal from the ANC is calculated for each reference signal. This results in a power spectrum for the values of $r$ that will have two peaks; one at $r=r_{v}$ and one at $r=r_{a}$ where the latter is the desired ratio. The implementation of DST used for this paper uses a recursive least squares (RLS) adaptive FIR filter of order 128. It is found that this order gives a desirable performance and increasing the order will only slow down the algorithm. The coefficient $r$ is increased by a step size of 0.01 to ensure the resolution in $\mathrm{SpO}_{2}$ is less than $1 \%$.

\section{INDEPENDENT COMPONENT ANALYSIS}

The general ICA model is formulated as

$$
\mathbf{X}=\mathbf{A S}
$$

where $\mathbf{X}$ is a matrix of observed multiple signal samples, $\mathbf{A}$ is a mixing matrix, and $\mathbf{S}$ is a matrix of independent source signal samples. The aim is then to estimate $\mathbf{A}$ and $\mathbf{S}$.

By assuming each of the pulse oximetry recordings contains a component representing the PPG signal $s_{1}(t)$ and an independent noise component $s_{2}(t)$ the ICA model for pulse oximetry is given by the 2 -by-2 linear mixing system ${ }^{1}$

$$
\begin{aligned}
x_{r}(t) & =a_{11} s_{1}(t)+a_{12} s_{2}(t) \\
x_{i r}(t) & =a_{21} s_{1}(t)+a_{22} s_{2}(t)
\end{aligned}
$$

where $a_{i j}$ are the coefficients determining the elements of the mixing matrix $\mathbf{A}$ in (6). The optical ratio is then estimated by the ratio of the mixing coefficients of the PPG source signal

$$
R=\frac{a_{11}}{a_{21}}
$$

\section{A. FastICA}

FastICA works by maximizing the negentropy of the independent source signals. Negentropy can be interpreted as a measure of non-Gaussianity ${ }^{2}$. The main advantage of this algorithm is due to its simple fixed-point iteration scheme.

\section{B. Maximum Likelihood ICA}

ICAML separates the independent identically distributed source signals by maximizing the likelihood of the mixing matrix. To use the maximum likelihood approach the score function associated with the source prior distribution is required.

\footnotetext{
${ }^{1}$ An extended model is provided in (10). See also the future work section below.

${ }^{2}$ It is well-known that white Gaussian distributed source signals can not be separated, [19].
} 


\section{Molgedey and Schouster ICA}

ICAMS is based on dynamic decorrelation and requires that the sources have different autocorrelation functions. The mixing matrix is estimated by maximizing the difference in autocorrelation of the source signals.

\section{Mean Field ICA}

ICAMF is a Bayseian probabilistic method for solving the ICA problem and is rather flexible with respect to specification of constraints and prior assumptions. The model differs from the general ICA model as it assumes the observed signals $\mathbf{X}$ are generated with an additive white noise source E, thus

$$
\mathbf{X}=\mathbf{A} \mathbf{S}+\mathbf{E}
$$

In the present context this is a reasonable assumption since it is unrealistic that the noise signals picked up in the red and infrared channels are identical except for scaling, as assumed in (7). The noise $\mathbf{E}$ is assumed to be Gaussian with zero mean and covariance $\boldsymbol{\Sigma}$. The noise covariance matrix can further be constrained to be isotropic or diagonal for same noise variance on the observations or individual noise variance for each observation, respectively.

\section{Clinical Methods and Materials}

We perform non-invasive self testing on healthy subjects. The subject is seated at rest and the oxygen saturation measured on the third digit of the right hand by a pulse oximeter, Datex-Ohmeda AS/3 Compact Patient Monitor, Pulse Oximeter. The Electronic Patch is placed on the skin over the third digit of the left hand. The PPG signals are measured continuously and the reference $\mathrm{S}_{\mathrm{p}} \mathrm{O}_{2}$ value is read of every 30s. The sampling frequency of the PPG data is $f_{s}=200 \mathrm{~Hz}$. The total length of the PPG sequences for the three subjects and the starting and ending reference $\mathrm{S}_{\mathrm{p}} \mathrm{O}_{2}$ levels are given in Table I.

The subjects re-breath in a closed circuit in order to lower the oxygen saturation as illustrated in Fig. 3. At the initiation of each test the circuit is filled with approximately 50 - 70 liters of atmospheric air. Carbon dioxide $\mathrm{CO}_{2}$ is absorbed from the circuit by exhaling through a filter of calcium hydroxide, Soda Lime (Dräger). The measurements are initiated by a few deep inspirations of $100 \%$ oxygen and then the breathing is changed to the closed circuit. The subject now breath continuously in the closed circuit. A decline in oxygen saturation is measured as the oxygen concentration in the circuit decreases.

Prior to the investigations it is agreed to end the testing when the oxygen saturation reached $75 \%$ or earlier if the subject felt uncomfortable.

\section{Signal Processing}

The optical ratio $R$ is calculated for signal windows of 30 s corresponding to the reference measurements. For each window the signal processing described in the following is applied.

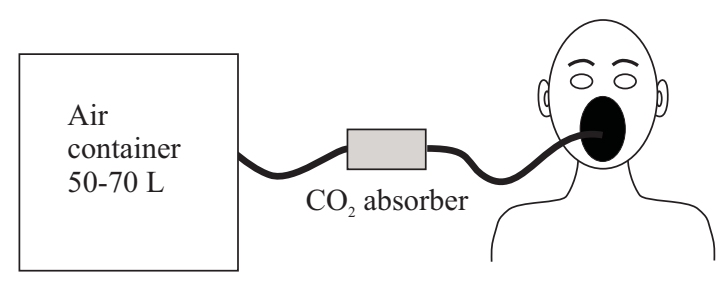

Fig. 3. To lower the oxygen saturation a closed circuit is used. The subjects re-breath air from a container through a carbon dioxide filter of calcium hydroxide, Soda Lime (Dräger). The $\mathrm{S}_{\mathrm{p}} \mathrm{O}_{2}$ is lowered from $100 \%$ to $75 \%$ on the average of $16 \mathrm{~min}$.

TABLE I

LENGTH OF SIGNALS AND START AND END REFERENCE $\mathrm{S}_{\mathrm{P}} \mathrm{O}_{2}$ LEVELS FOR THE THREE SUBJECTS.

\begin{tabular}{|c||c|c|c|}
\hline Subject & Length (s) & $\mathrm{S}_{\mathrm{p}} \mathrm{O}_{2}$ start (\%) & $\mathrm{S}_{\mathrm{p}} \mathrm{O}_{2}$ end (\%) \\
\hline 1 & 760 & 98 & 74 \\
2 & 1111 & 100 & 86 \\
3 & 1020 & 100 & 70 \\
\hline
\end{tabular}

\section{A. Pre-processing and estimation of $R$}

The PPG signals are normalized by subtracting the DCcomponent and dividing by the DC-component. This is done to scale the recordings from each subject to the same range. To avoid high frequency electrical noise and low frequency noise components bandpass filtering is applied. It is found that the best performance is achieved by a 8th order IIR (Butterworth) filter. The cutoff frequencies are optimized for each algorithm by evaluating the estimates for range of cutoff frequencies using the evaluation scheme explained in the following section. The cutoff frequencies resulting in smallest error for each algorithm is then chosen. The cutoff frequencies are given in Table II as lower cutoff frequency $f_{l}$ and higher cutoff frequency $f_{h}$. To avoid distortion due to filter settling time an overlap of $4 \mathrm{~s}$ is added to the front of the signal windows before filtering and removed again after filtering. After normalization and bandpass filtering $R$ or $r_{a}$ is estimated by ICA or DST respectively.

\section{B. Evaluation Scheme}

To evaluate the estimates of $R$ a calibration curve that relates the $R$-values to the $\mathrm{S}_{\mathrm{p}} \mathrm{O}_{2}$ in $\%$ is needed. From the theory of pulse-oximetry [20] it is known that the calibration curve is on the form

$$
\mathrm{S}_{\mathrm{p}} \mathrm{O}_{2}=\frac{a+b R}{c+d R} .
$$

However, in the important $\mathrm{S}_{\mathrm{p}} \mathrm{O}_{2}$ range from $70 \%$ to $100 \%$ a linear approximation to the calibration curve can be used. It follows from derivation using Beer-Lambert's law that the gradient of the linear approximation should be negative [20].

To obtain a calibration curve that does not depend heavily on the available data set a training and test set evaluation scheme is applied: The $R$-estimates are divided into a training and test set consisting of values from two and one subjects respectively. A calibration curve is calculated from the training set by using the leave-one-out method. One estimate is left out and a linear model is fitted to the rest 
TABLE II

BANDPASS FILTER CUT-FREQUENCIES

\begin{tabular}{|l||c|c|}
\hline Algorithm & $f_{l}(\mathrm{~Hz})$ & $f_{h}(\mathrm{~Hz})$ \\
\hline DST & 0.9 & 3 \\
FastICA & 1 & 3 \\
ICAML & 1 & 2 \\
ICAMS & 1 & 3 \\
ICAMF & 0.8 & 3 \\
\hline
\end{tabular}

of the estimates. This is repeated for all estimates in the training set and the mean of the coefficients of all fitted calibration curves is used as a final linear calibration curve for testing. The obtained calibration curve is used to predict test set $\mathrm{S}_{\mathrm{p}} \mathrm{O}_{2}$ values from $R$-values. Finally, the test error is calculated as the Euclidean difference between the estimated and the reference measurement of $\mathrm{S}_{\mathrm{p}} \mathrm{O}_{2}$. The procedure is repeated for all three combinations of training and test set. The average error is then calculated for each algorithm.

The signal processing can be summarized into five steps:

1) Normalization

2) Bandpass filtering

3) Estimation of $R$ or $r_{a}$

4) Estimation of calibration curve

5) Estimation of $\mathrm{S}_{\mathrm{p}} \mathrm{O}_{2}$

\section{RESULTS}

In Fig. 4 is shown the result for the DST algorithm as the reference $\mathrm{S}_{\mathrm{p}} \mathrm{O}_{2}$ values versus the estimated arterial ratios, $r_{a}$. It is seen from this graph that the algorithm gives a calibration curve and that the results from the three subjects fall within the same range. The average error is found to be $3.0 \%$.

In Fig. 5, 6, 7, and 8 are shown the results for the four ICA algorithms. From Fig. 5 it is seen that few estimates are available as the FastICA algorithm fails to converge for parts of data. The estimated calibration curve is therefor weaker than what is obtained with DST. The ICAML, ICAMS, and ICAMF algorithms all give a correct calibration curve. The ICAMS and ICAMF algorithms give results that are more similar to the result from the DST algorithm compared to the ICAML algorithm. For the DST, ICAMS, and ICAMF the linear fit is correct in the range $100 \%-80 \%$ but fails in the range $80 \%-70 \%$.

ICAMF allows one to use several prior distributions for the sources and we found that a bi-Gauss distribution is the best choice for the PPG-signals. The mixing matrix is constraint to be positive as $R$ should be positive. Further, we found that a configuration with a diagonal covariance matrix for the noise term gave the best results.

The average Euclidean error and the relative error compared to the DST error are listed in Table III for all algorithms. With respect to these measures it is seen that FastICA and ICAML performs $27 \%$ and $13 \%$ worse than DST, respectively. Both ICAMS and ICAMF performs better than DST with relative improvements of $3 \%$ and $13 \%$, respectively.

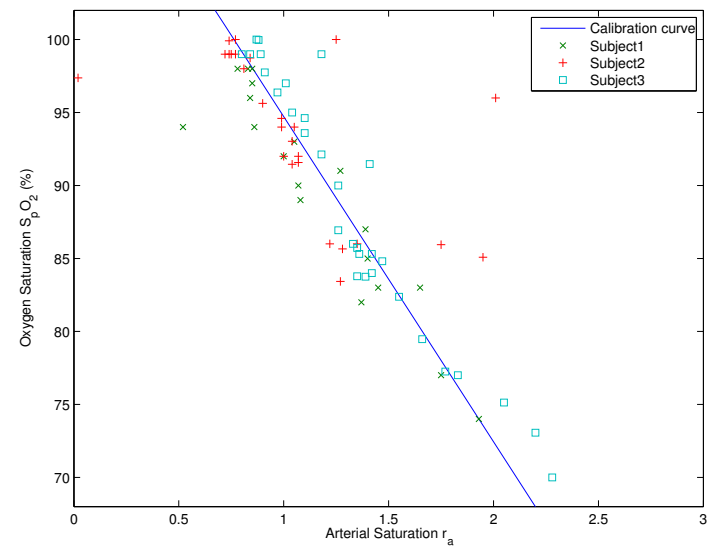

Fig. 4. Calibration curve and estimates obtained using DST. It is seen that the linear model fits most of the estimates, but some estimates could be considered as outliers.

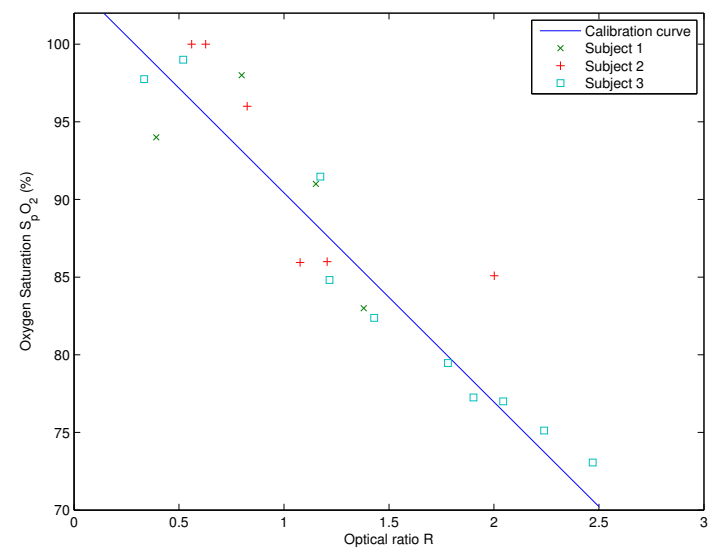

Fig. 5. Calibration curve obtained using FastICA. It should be noted that a fewer estimates are seen. This is due to the algorithm not converging for parts of the data.

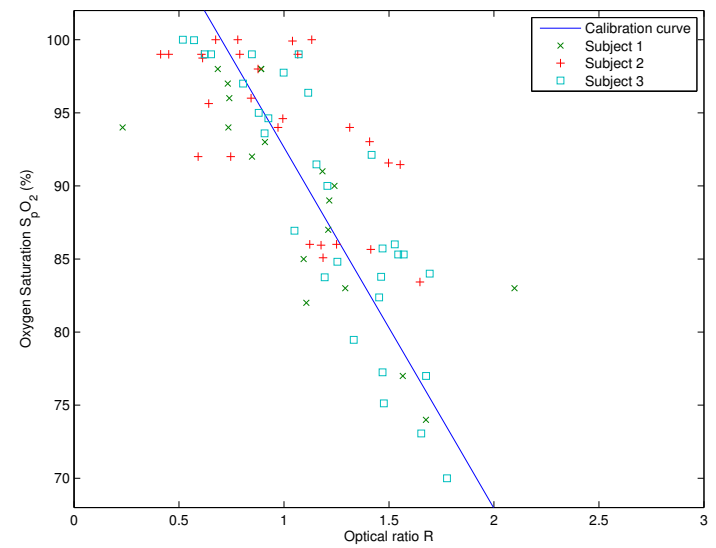

Fig. 6. Calibration curve and estimates obtained using ICAML. Compared to the DST algorithm the estimates deviates more from the calibration curve. 


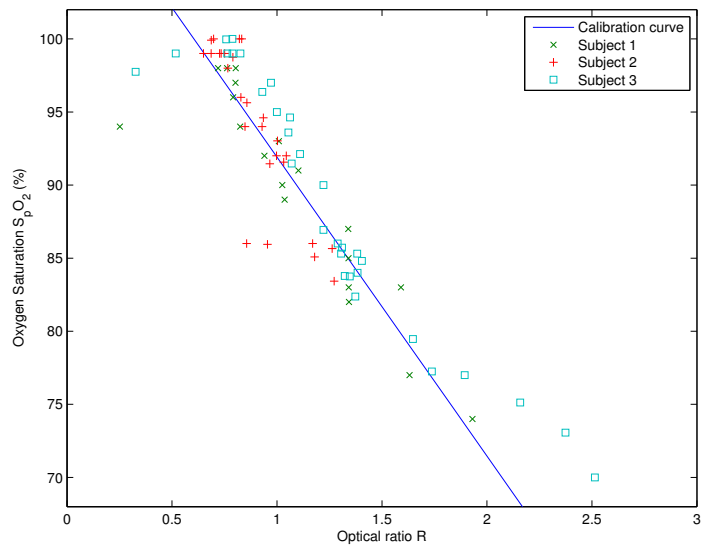

Fig. 7. Calibration curve and estimates obtained using ICAMS. The performance is similar to the DST algorithm. Notice the estimates below $80 \%$ do not fit the linear model approximation.

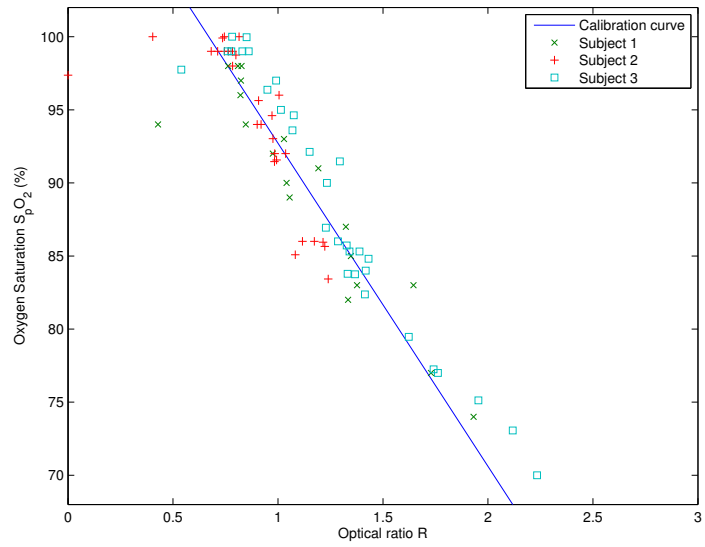

Fig. 8. Calibration curve and estimates obtained using ICAMF. The performance is similar to the DST algorithm, but the algorithm appears to be more robust when considering possible outliers.

\section{DISCUSSION}

The purpose of this work is to study ICA algorithms by comparing them with respect to each other and to the DST algorithm. The purpose is not to obtain a calibration curve for future use. Therefore the quality of the reference data is of less importance and we choose to use a non-invasive method that does not require a clinical protocol (by Danish law). More precise reference data could be obtained by collecting arterial blood samples from which the oxygen saturation can be found accurately by a blood gas analyzer.

The non-invasive method we have used resulted in an error of $3.0 \%$ for the DST algorithm. A commercial pulse

TABLE III

MEAN ERROR FOR EACH ALGORITHM AND ERROR RELATIVE TO DST.

\begin{tabular}{|l||c|c|}
\hline Algorithm & $\mathrm{S}_{\mathrm{p}} \mathrm{O}_{2}$ error & Error relative to DST \\
\hline DST & 3.0 & - \\
FastICA & 3.8 & $27 \%$ \\
ICAML & 3.4 & $13 \%$ \\
ICAMS & 2.9 & $-3 \%$ \\
ICAMF & 2.6 & $-13 \%$ \\
\hline
\end{tabular}

oximeter by Masimo Corporation has a specified accuracy of $2 \%$ [21], the obtained reference data must therefore be considered useful.

The obtained calibration curves suggest that the linear model approximation is good in the $\mathrm{S}_{\mathrm{p}} \mathrm{O}_{2}$ range of $80 \%$ to $100 \%$ whereas below this range a nonlinear relation is a better choice.

\section{CONCLUSION AND FUtURE WORK}

\section{A. Conclusions}

We have successfully demonstrated ICA algorithms in the application of estimating the arterial oxygen saturation by pulse oximetry. Four ICA algorithms have been evaluated and compared to DST. The four algorithms did perform differently. The ICAMF is found to give the best results with an relative improvement over the DST algorithm of $13 \%$. The FastICA algorithm failed to converge for parts of the data and showed a decline of $27 \%$ compared to DST. The ICAMS showed a relative improvement of $3 \%$ over DST and the ICAML showed a decline of $13 \%$ compared to DST.

It can be concluded that ICA can be used for calculating $\mathrm{S}_{\mathrm{p}} \mathrm{O}_{2}$ when the ICA problem is solved using the right constraints. We conclude that the difference in autocorrelation of the source signals and the bi-Gaussian distribution of the PPG signal and individual noise on the red and infrared recordings are the useful constraints.

The accuracy of the $\mathrm{S}_{\mathrm{p}} \mathrm{O}_{2}$ values do indeed depend on the choice of ICA algorithm, and we have found that the ICAMF is the best choice.

\section{B. Future Work}

Motion artifacts are serious noise component in the recorded signals. We believe that ICA models could provide a good framework for handling such problems which is suggested by initial experiments. We consider a more general framework using a linear state-space based model suggested in [22] which allows for more flexible mixing and prior source models. In addition, the model can handle the socalled underdetermined case, i.e. more source signals than recorded signals. This is useful for modeling different noise sources which could have very different dynamics.

\section{ACKNOWLEDGEMENTS}

We would like to acknowledge our collaborators within the Danish innovation consortium - "The Electronic Patch".

\section{REFERENCES}

[1] R. G. Haahr, S. Duun, K. Birkelund, P. Raahauge, P. Petersen, H. Dam, L. Nørgaard, E. V. Thomsen, "A Novel Photodiode for Reflectance Pulse Oximetry in low-power applications" Conf Proc IEEE Eng Med Biol Soc, pp. 2350 - 2353, 2007

[2] R. G. Haahr, S. Duun, E. V. Thomsen, K. Hoppe, and J. Branebjerg, "A Wearable "Electronic Patch" for Wireless Continuous Monitoring of Chronically Diseased Patients", Proc. IEEE BSN 2008 conf., pp. 66-70, 2008

[3] S. Duun, R. G. Haahr, K. Birkelund, P. Raahauge, P. Petersen, H. Dam, L. Nørgaard, E. V. Thomsen, "A Novel Ring Shaped Photodiode for refelctance Pulse Oximetry in Wireless Applications" Conf Proc IEEE Sensors, pp. 596 - 599, 2007 
[4] J. M. Goldman, M. T. Petterson, R. J. Kopotic, and S. J. Barker, "Masimo signal extraction pulse oximetry," Journal of Clinical Monitoring and Computing, vol. 16, pp. 475-483, 2000.

[5] Masimo Corporation, 40 Parker, Irvine, CA 92618 USA.

[6] B. S. Kim el. al, "Motion Artifact Reduction in Photoplethysmography Using Independent Component Analysis", IEEE Trans Biomed Eng. , vol. 53, no. 3, pp. 566-568, 2006.

[7] J. Yao el. al, "A Short Study to Assess the Potential of independent Component Analysis for Motion Artifact Separation in Wearable Pulse Oximeter Signals", Conf. Proc. IEEE Eng. Med. Biol. Soc, pp. 3585$3588,2005$.

[8] http://isp.imm.dtu.dk/pulseoximetry/

[9] A. Hyvarinen, "Fast and robust fixed-point algorithms for independent component analysis," IEEE Transactions on Neural Networks, vol. 10, pp. 626-634, 1999.

[10] P.F. Stetson, Blind scource separation of pulse oximetry signals, 2004, Nellcor Puritan Bennett Inc.:U.S. patent no. 7,079,880 B2.

[11] A. Bell and T. Sejnowski, "An information-maximization approach to blind separation and blind deconvolution," Neural Computation, vol. 7, pp. 1129-1159, 1995.

[12] L. K. Hansen, J. Larsen, and T. Kolenda, "Blind detection of independent dynamic components," In proc. IEEE ICASSP'2001, vol. 5, pp. 3197-3200, 2001.

[13] H. Nielsen, "UCMINF - an algorithm for unconstrained, nonlinear optimization,” IMM, Technical University of Denmark, Tech. Rep. IMM-TEC-0019, 2001.

[14] L. Molgedey and H. Schuster, "Separation of independent signals using time-delayed correlations," Physical Review Letters, vol. 72 no. 23, pp. 3634-3637, 1994.

[15] L. K. Hansen, J. Larsen, and T. Kolenda, "On Independent Component Analysis for Multimedia Signals," in Multimedia Image and Video Processing, pp. 175-199, CRC Press, 2000.

[16] P. Højen-Sørensen, O. Winther and L K. Hansen, "Mean Field Approaches to Independent Component Analysis," Neural Computation, vol. 14, pp. 889-918, 2002.

[17] O. Winther and K. B. Petersen, "Flexible and efficient implementations of bayesian independent component analysis," Neurocomputing, vol. 71, pp. 221-233, 2007.

[18] B. Widrow el. al, "Adaptive noise cancelling: Principles and applications" Proceedings of the IEEE, vol. 63, no. 12, pp. 1692-1716, 1975.

[19] D. J. C. MacKay, Information Theory, Inference, and Learning Algorithms. Cambridge University Press, 2007, ch. 34.

[20] J. G. Webster, Ed., Design of Pulse Oximeters. Institute of Physics Publishing, 1997, ch. 4.

[21] Masimo Corporation, "Rad-9 signal extraction pulse oximeter"

[22] R. K. Olsson and L. K. Hansen, "Linear state-space models for blind source separation," Journal of Machine Learning Research, vol. 7, pp. 2585-2602, 2006. 